\title{
SYMBOLIC POWERS OF GENERALIZED STAR CONFIGURATIONS OF HYPERSURFACES
}

\author{
KUEI-NUAN LIN AND YI-HUANG SHEN
}

\begin{abstract}
We introduce the class of sparse symmetric shifted monomial ideals. These ideals have linear quotients and their Betti numbers are computed. Using this, we prove that the symbolic powers of the generalized star configuration ideal are sequentially Cohen-Macaulay under some mild genericness assumption. With respect to these symbolic powers, we also consider the Harbourne-Huneke containment problem and establish the Demailly-like bound.
\end{abstract}

\section{INTRODUCTION}

In the simplest case, a star configuration of codimension $c$ in $\mathbb{P}^{n}$ is a certain union of linear subspaces $V_{1}, \ldots, V_{p}$ each of codimension $c$. The study of various aspects of generalizations of star configurations has attracted considerable attention recently; see for example, [6], [7], [11], [12], [17], [18] and [19], to name just a few. As a sequel to our previous work [17] along this line, we continue to investigate the symbolic powers of the generalized star configuration of hypersurfaces.

Let $R=\mathbb{K}\left[x_{0}, \ldots, x_{n}\right]$ be a standard graded polynomial ring over an infinite $\mathbb{K}$ and denote its graded maximal ideal by $\mathfrak{m}$. Suppose that $s \geq n+1$ and let $\mathcal{F}=\left\{f_{1}, \ldots, f_{s}\right\}$ be a set of forms in $R$ satisfying some genericness condition. For a fixed choice of positive integers $a$ and $b$ such that $a \leq b s$, the generalized star configuration ideal that we shall study here is the uniform a-fold product ideal $I_{a}\left(\mathcal{F}^{b}\right):=I_{a}\left(f_{1}^{b} \cdots f_{s}^{b}\right)$.

In general, the $a$-fold product ideal $I_{a}\left(f_{1}^{b_{1}} \cdots f_{s}^{b_{s}}\right)$ is generated by the $a$-fold products of the forms $f_{1}, \ldots, f_{s}$ with multiplicities $b_{1}, \ldots, b_{s}$ respectively:

$$
\left\langle f_{1}^{n_{1}} \cdots f_{s}^{n_{s}}: 0 \leq n_{i} \leq b_{i} \text { for each } i \text { such that } \sum_{i} n_{i}=a\right\rangle .
$$

It has also been studied extensively recently; see for example [2] and [10]. This notion was originally introduced as a nice tool for determining the minimum distance of linear codes in the coding theory. And it also emerges naturally when dealing with the higher order Orlik-Terao algebra of hyperplane arrangements.

The $a$-fold product ideal $I_{a}\left(f_{1}^{b_{1}} \cdots f_{s}^{b_{s}}\right)$ is uniform when the multiplicities $b_{1}, \ldots, b_{s}$ are all identical, say, to some positive integer $b$. Whence, we will write it as $I_{a}\left(\mathcal{F}^{b}\right)$ for simplicity. It is treated as the generalized star configuration ideal, since when $b=1$, we will get back the star configuration ideal of hypersurfaces

$$
I_{c, \mathcal{F}}:=\bigcap_{1 \leq i_{1}<\cdots<i_{c} \leq s}\left\langle f_{i_{1}}, \ldots, f_{i_{c}}\right\rangle
$$

for $c=s-a+1$, which were studied for instance in [12] and [18]. In their work, the set $\mathcal{F}$ is often assumed to be $c$-generic, namely any subset of size at most $c+1$ will form a regular sequence.

2020 Mathematics Subject Classification. 13A15, 13A50, 13D02, 14N20, 52C35.

Keyword: Betti numbers, linear quotients, star configuration, symbolic power, containment problem, stable Harbourne-Huneke conjecture. 
Our current work will focus on the study of the symbolic powers of the generalized star configuration ideal $I_{a}\left(\mathcal{F}^{b}\right)$. Recall that give an ideal $I$ in a ring $R$, the $m$-th symbolic power of $I$ is defined by

$$
I^{(m)}:=\bigcap_{\mathfrak{p} \in \operatorname{Ass}(R / I)} I^{m} R_{\mathfrak{p}} \cap R .
$$

For instance, when $I$ is the defining ideal of a reduced affine scheme over an algebraically closed field of characteristic zero, Zariski and Nagata showed that $I^{(m)}$ is generated by the polynomials whose partial derivatives of orders up to $m-1$ vanish on this scheme. And in the previous work [17], we have already studied the resurgence and symbolic defect of $I_{a}\left(\mathcal{F}^{b}\right)$, as well as the Betti table and the primary decomposition of its symbolic powers.

In 11] and [12, Geramita, Harbourne, Migliore, and Nagel showed that the symbolic powers of the ordinary star configuration ideal define arithmetically Cohen-Macaulay subschemes. It is then natural to ask if the same property still holds in the generalized case. Notice that the ideal $I_{a}\left(\mathcal{F}^{b}\right)$ is no longer unmixed for $b \geq 2$. Thus, the correct question can be put as: whether the symbolic powers of this ideal are all sequentially Cohen-Macaulay? We provide a positive answer under some mild genericness assumption.

In this work, we also study the Harbourne-Huneke containment problem, which was originally raised in [14]. After [4], a generalized version can be stated as: given a homogeneous ideal $I$ of big height $h$ in a standard graded ring with the maximal homogeneous ideal $\mathfrak{m}$, does the inequality

$$
I^{(\ell(h+m-1))} \subseteq \mathfrak{m}^{\ell(h-1)}\left(I^{(m)}\right)^{\ell}
$$

hold for all $m, \ell \geq 1$ ? The comparison between ordinary powers of an ideal and its symbolic ideals is wildly open in general; see, for example, [5] and [16]. We provide a positive answer for Harbourne-Huneke containment problem in the case $I=I_{a}\left(\mathcal{F}^{b}\right)$ under some mild genericness assumption. As a by-product, we establish the Demailly-like bound in this case as well.

As usual, for a homogeneous ideal $J$, we will write $\alpha(J)$ for the least degree of nonzero forms in this ideal. Then, our main results can be summarized in the following.

Theorem 1.1. Let $a$ and $b$ be positive integers such that $(b-1) s+1<a \leq b s$. We assume further that $n \geq b s-a+1$ and $\mathcal{F}=\left\{f_{1}, \ldots, f_{s}\right\}$ is a set of $(b s-a+1)$-generic $d$-forms in $R=\mathbb{K}\left[x_{0}, \ldots, x_{n}\right]$. Then the following properties hold for the generalized star configuration ideal $I=I_{a}\left(\mathcal{F}^{b}\right)$ whose big height is known to be $h=b s-a+1$.

(a) The symbolic powers of I are all sequentially Cohen-Macaulay.

(b) The ideal I satisfies

$$
I^{(\ell(h+m-1)-h+k)} \subseteq \mathfrak{m}^{d((\ell-1)(h-1)+k-1)(b h-(b s-a))}\left(I^{(m)}\right)^{\ell}
$$

for all positive integers $k, \ell$, and $m$.

(c) The inequality

$$
\frac{\alpha\left(I^{(\ell)}\right)}{\ell} \geq \frac{\alpha\left(I^{(m)}\right)+h-1}{m+h-1}
$$

holds for all $\ell, m \geq 1$.

\section{SPARSE SYMmetriC SHIFTED IDEALS}

To show the sequentially Cohen-Macaulayness of the symbolic powers of the generalized star configuration ideal $I_{a}\left(\mathcal{F}^{b}\right)$, we have to introduce first the sparse symmetric shifted ideals. These squarefree monomial ideals can be viewed as variations of the symmetric shifted ideals introduced in [3]. Likewise, the new ideals have linear quotients 
(Theorem 2.6) and the Betti tables can be obtained combinatorially with ease (Corollary 2.18).

2.1. Definition and the linear quotient property. The ground ring that we shall consider is the polynomial $\operatorname{ring} Z=\mathbb{K}\left[z_{i, j}: 1 \leq i \leq s, 1 \leq j \leq b\right]$ over an arbitrary field $\mathbb{K}$. For each tuple $\boldsymbol{\mu}=\left(\mu_{1}, \ldots, \mu_{s}\right) \in\{0,1,2, \ldots, b\}^{s}$, a monomial of the form $z^{\mu}:=\prod_{i=1}^{s} z_{i, \mu_{i}}$ will be called a sparse squarefree monomial. Here, when $\mu_{i}=0, z_{i, \mu_{i}}$ is understood to be the identity element $1 \in Z$.

Recall that a tuple $\boldsymbol{\lambda}=\left(\lambda_{1}, \ldots, \lambda_{s}\right) \in \mathbb{Z}_{>0}^{s}$ is called a partition of length $s$, if $\lambda_{1} \leq$ $\cdots \leq \lambda_{s}$. For the sparse squarefree monomial $f=z^{\mu}$ above, we may suppose that the multi-set

Now, we write

$$
\left\{\mu_{1}, \ldots, \mu_{s}\right\}=\left\{\lambda_{1} \leq \cdots \leq \lambda_{s}\right\}
$$

$$
\boldsymbol{\lambda}(f):=\left(\lambda_{1}, \ldots, \lambda_{s}\right) \in \mathbb{Z}_{\geq 0}^{s},
$$

and call it the partition associated to $f$. If $\boldsymbol{\lambda}(f)=\left(0, \ldots, 0, e_{1}, \ldots, e_{d}\right)$ where $e_{1} \geq 1$, then the support degree of both $f$ and $\boldsymbol{\lambda}=\boldsymbol{\lambda}(f)$ are

$$
\operatorname{suppdeg}(f)=\operatorname{suppdeg}(\boldsymbol{\lambda}):=d,
$$

and the weight of both $f$ and $\boldsymbol{\lambda}$ are

$$
w(f)=|\boldsymbol{\lambda}|:=e_{1}+\cdots+e_{d}=\lambda_{1}+\cdots+\lambda_{s}=\mu_{1}+\cdots+\mu_{s} .
$$

Related, a sparse squarefree monomial in $Z$ whose support degree is $s$, is called a rainbow monomial in [1, Subsection 1.3].

Let $\boldsymbol{\mu}^{\prime}=\left(\mu_{1}^{\prime}, \ldots, \mu_{s}^{\prime}\right) \in\{0,1,2, \ldots, b\}^{s}$ be another tuple. We will write $\boldsymbol{\mu}^{\prime} \leq_{0} \boldsymbol{\mu}$ if

- $\mu_{i}^{\prime} \leq \mu_{i}$ for each $i$, and

- $\mu_{i}^{\prime}=0$ if and only if $\mu_{i}=0$.

Notice that the permutation group $\mathfrak{S}_{s}$ has an action on $Z$, such that for each permutation $\sigma \in \mathfrak{S}_{s}$ and a variable $z_{i, j}$ in $Z$, we have $\sigma\left(z_{i, j}\right)=z_{\sigma(i), j}$.

Definition 2.1. A squarefree monomial proper ideal $J$ of $Z$ will be called sparse symmetric shifted if it satisfies the following requirements.

(S-1) The ideal $J$ is generated by some sparse squarefree monomials.

(S-2) For each minimal monomial generator $f=\boldsymbol{z}^{\boldsymbol{\mu}} \in G(J)$ with $\boldsymbol{\mu}=\left(\mu_{1}, \ldots, \mu_{s}\right)$, if $f^{\prime}=\boldsymbol{z}^{\boldsymbol{\mu}^{\prime}}$ with $\boldsymbol{\mu}^{\prime}=\left(\mu_{1}^{\prime}, \ldots, \mu_{s}^{\prime}\right)$ and $\boldsymbol{\mu}^{\prime} \leq_{0} \boldsymbol{\mu}$, then $f^{\prime} \in J$.

(S-3) The ideal $J$ is invariant under the action of $\mathfrak{S}_{s}$.

Remark 2.2. We may imagine the variables in $Z$ displayed in the canonical matrix $\boldsymbol{Z}=\left(z_{i, j}\right)_{s \times b}$. Then, roughly speaking, the sparse symmetry shifted ideal above has sparseness, vertical (upward) shiftiness, and horizontal symmetry. And these properties correspond to the (S-1), (S-2), and (S-3) respectively. Meanwhile, the symmetric shifted ideal, introduced in [3], has both horizontal shiftiness and horizontal symmetry, since it lacks the sparse structure here.

Lemma 2.3. Let $J$ be a sparse symmetric shifted ideal in $Z$. Suppose that there is some sparse squarefree monomial $f=\prod_{i} z_{\sigma(i), \mu_{i}} \in J$ for some $\sigma \in \mathfrak{S}_{s}$. If $f^{\prime}=\prod_{i} z_{\sigma^{\prime}(i), \mu_{i}^{\prime}}$ for some $\sigma^{\prime} \in \mathfrak{S}_{s}$ with $\left(\mu_{1}^{\prime}, \ldots, \mu_{s}^{\prime}\right) \leq_{0}\left(\mu_{1}, \ldots, \mu_{s}\right)$, then $f^{\prime} \in J$ as well.

Proof. By the symmetry condition (S-3), it suffices to consider the case when $\sigma=\sigma^{\prime}=$ id $\in \mathfrak{S}_{s}$. Since $f \in J$, there exists some minimal monomial generator $g=\prod_{i} z_{i, \tau_{i}} \in G(J)$ such that $\tau_{i}=\mu_{i}$ or 0 for each $i$. Define $\tau_{i}^{\prime}=\min \left(\tau_{i}, \mu_{i}^{\prime}\right)$ for each $i$. Since $\left(\tau_{1}^{\prime}, \ldots, \tau_{s}^{\prime}\right) \leq_{0}$ $\left(\tau_{1}, \ldots, \tau_{s}\right)$, the monomial $g^{\prime}:=\prod_{i} z_{i, \tau_{i}^{\prime}} \in J$ by the shiftiness condition (S-2). As $g^{\prime}$ divides $f^{\prime}$, this implies that $f^{\prime} \in J$ as well. 
Corollary 2.4. Let $J$ be a sparse symmetric shifted ideal in $Z$. Suppose that $f=$ $\prod_{i} z_{\sigma(i), \lambda_{i}} \in J$ where $\boldsymbol{\lambda}(f)=\left(\lambda_{1}, \ldots, \lambda_{s}\right)$ and $\sigma \in \mathfrak{S}_{s}$. If $\lambda_{i_{1}}=0<k \leq \lambda_{i_{2}}$ for some $i_{1}$ and $i_{2}$, then $f^{\prime}:=f z_{\sigma\left(i_{1}\right), k} / z_{\sigma\left(i_{2}\right), \lambda_{i_{2}}} \in J$.

Remark 2.5. Suppose that the ideal $J \subset Z$ is generated by some sparse squarefree monomials. If $f$ is a monomial in $Z$, then the colon ideal $J: Z f$ is also generated by some sparse squarefree monomials that are coprime to $f$. To see this, it suffices to notice that when $g$ is a sparse squarefree monomial, then $\langle g\rangle: f$ is generated by the sparse squarefree monomial $g / \operatorname{gcd}(f, g)$.

Here is the first main result of this section.

Theorem 2.6. If $J$ is a sparse symmetric shifted ideal in $Z$, then it has linear quotients.

Proof. Inspired by the treatment in [3], we first consider the lexicographic ordering $>_{\text {lex }}$ on $Z$ where the variables $z_{i, j}>z_{i^{\prime}, j^{\prime}}$ if and only if

- $j<j^{\prime}$, or

- $j=j^{\prime}$ and $i<i^{\prime}$.

Thus, among those squarefree monomials of degree $s, z_{1,1} z_{2,1} \cdots z_{s, 1}$ is the initial (biggest) one with respect to $>_{\text {lex }}$.

Next, we take a total order $\prec$ on $\mathbb{Z}_{\geq 0}^{s}$ defined by

$$
\boldsymbol{a}=\left(a_{1}, \ldots, a_{s}\right) \prec \boldsymbol{b}=\left(b_{1}, \ldots, b_{s}\right)
$$

if and only if

- $\operatorname{suppdeg}(\boldsymbol{a})<\operatorname{suppdeg}(\boldsymbol{b})$, or

- $\operatorname{suppdeg}(\boldsymbol{a})=\operatorname{suppdeg}(\boldsymbol{b})$ and $|\boldsymbol{a}|<|\boldsymbol{b}|$, or

- $\operatorname{suppdeg}(\boldsymbol{a})=\operatorname{suppdeg}(\boldsymbol{b})$ and $|\boldsymbol{a}|=|\boldsymbol{b}|$, while the leftmost non-zero entry of $\left(a_{1}-b_{1}, \ldots, a_{s}-b_{s}\right)$ is negative.

Now, we are ready to introduce a total order on the minimal generating set $G(J)$, which, by abuse of notation, will still be denoted by $\prec$. For $f_{1}$ and $f_{2}$ in $G(J)$, we define $f_{1} \prec f_{2}$ if and only if

- $\boldsymbol{\lambda}\left(f_{1}\right) \prec \boldsymbol{\lambda}\left(f_{2}\right)$, or

- $\boldsymbol{\lambda}\left(f_{1}\right)=\boldsymbol{\lambda}\left(f_{2}\right)$ and $f_{1}>_{\text {lex }} f_{2}$.

Then, we sort the squarefree monomials in $G(J)$ with respect to $\prec$. To establish the linear quotient property, we take arbitrary minimal monomial generator $f \in G(J)$ and let

$$
J_{f}:=\langle g \in G(J): g \prec f\rangle .
$$

The remaining task is to show that the colon ideal $J_{f}:_{Z} f$ is linear for each such $f$. We can accomplish this task with the following two steps.

(i) First of all, we describe explicitly the set $\mathcal{G}_{f}$ of variables that belong to this colon ideal.

(ii) Next, we prove that the set $\mathcal{G}_{f}$ actually generates the colon ideal $J_{f}: f$ in general. Thus, $J_{f}: f$ is linear, as expected.

They are established in the coming Lemma 2.7 and Lemma 2.8 respectively. And consequently, the ideal $J$ has linear quotients.

In the remaining of this subsection, we fix a monomial $f$ in $G(J)$. The first task is to describe the set $\mathcal{G}_{f}$ which collects the variables in the colon ideal $J_{f}: f$. Suppose that the partition $\boldsymbol{\lambda}(f)=\left(\lambda_{1}, \ldots, \lambda_{s}\right)$. Now, there is some permutation $\sigma \in \mathfrak{S}_{s}$ such that $f=\prod_{i=1}^{s} z_{\sigma(i), \lambda_{i}}$. Recall that for $i$ with $\lambda_{i}=0, z_{\sigma(i), \lambda_{i}}$ is understood to be the identity element $1 \in Z$. And by abuse of notation, we define $\max (f):=\max \left\{\sigma(k): \lambda_{k}=\lambda_{s}\right\}$. 
Lemma 2.7. The set $\mathcal{G}_{f}$ is the disjoint union of the following three types of sets:

- $\left\{z_{\sigma(i), j}: 1 \leq j<\lambda_{i}\right.$ and $\left.\lambda_{i}>0\right\}$,

- $\left\{z_{\sigma(i), j}: 1 \leq j<\lambda_{s}\right.$ and $\left.\lambda_{i}=0\right\}$, and

- $\left\{z_{\sigma(i), \lambda_{s}}: \sigma(i)<\max (f)\right.$ and $\left.\lambda_{i}=0\right\}$.

A quick example illustrating the linear generating set $\mathcal{G}_{f}$ above is given in Example 3.4 .

Proof. To confirm the assertion, roughly speaking, we have two cases.

(A) Firstly, we consider the case when $\lambda_{i}>0$. We will show that $z_{\sigma(i), j} \in \mathcal{G}_{f}$ if and only if $1 \leq j<\lambda_{i}$.

(i) When $1 \leq j<\lambda_{i}$, we look at the squarefree monomial $f^{\prime}:=f z_{\sigma(i), j} / z_{\sigma(i), \lambda_{i}}$. By (S-2), we have $f^{\prime} \in J$. Thus, we can find suitable $f^{\prime \prime} \in G(J)$ that divides $f^{\prime}$. Obviously, we have $\operatorname{suppdeg}\left(f^{\prime \prime}\right) \leq \operatorname{suppdeg}\left(f^{\prime}\right)=\operatorname{suppdeg}(f)$ and $w\left(f^{\prime \prime}\right) \leq w\left(f^{\prime}\right)<w(f)$. It follows that $f^{\prime \prime} \prec f$. Now, $z_{\sigma(i), j} f \in\left\langle f^{\prime}\right\rangle \subseteq$ $\left\langle f^{\prime \prime}\right\rangle \subseteq J_{f}$. Consequently, $z_{\sigma(i), j} \in J_{f}: f$, as expected.

(ii) Suppose that $j=\lambda_{i}$. It follows from Remark 2.5 that $z_{\sigma(i), \lambda_{i}} \notin \mathcal{G}_{f}$.

(iii) When $j>\lambda_{i}$, we suppose for contradiction that $z_{\sigma(i), j} \in \mathcal{G}_{f}$. Since now $f z_{\sigma(i), j} \in J_{f}$, there exists some $f^{\prime \prime} \in G\left(J_{f}\right)$ such that $f^{\prime \prime}$ divides $f z_{\sigma(i), j}$. If $z_{\sigma(i), j}$ does not divide this $f^{\prime \prime}$, then $f^{\prime \prime}$ divides $f$ directly, contradicting the minimality of $f$. Thus, $z_{\sigma(i), j}$ divides $f^{\prime \prime}$. Since $f^{\prime \prime}$ is a sparse monomial, $z_{\sigma(i), \lambda_{i}}$ does not divide $f^{\prime \prime}$. Consequently, $f^{\prime \prime}$ divides the squarefree monomial $f^{\prime}:=f z_{\sigma(i), j} / z_{\sigma(i), \lambda_{i}}$. Now, $f^{\prime} \in J$ such that $\operatorname{suppdeg}\left(f^{\prime}\right)=\operatorname{suppdeg}(f)$ and $\left|\boldsymbol{\lambda}\left(f^{\prime}\right)\right|>|\boldsymbol{\lambda}(f)|$. Hence, we don't have $f^{\prime} \prec f$. Consequently, $f^{\prime \prime} \neq f^{\prime}$ and $\operatorname{suppdeg}\left(f^{\prime \prime}\right)<\operatorname{suppdeg}\left(f^{\prime}\right)=\operatorname{suppdeg}(f)$. Next, we look at $f^{\prime \prime \prime}:=$ $f^{\prime \prime} z_{\sigma(i), \lambda_{i}} / z_{\sigma(i), j}$, which belongs to $J$ by (S-2). Notice that $f^{\prime \prime \prime}$ properly divides $f$, as $f^{\prime \prime}$ properly divides $f^{\prime}$. This contradicts the minimality of $f$. Therefore, $z_{\sigma(i), j} \notin J_{f}: f$, as expected.

(B) Next, we consider the case when $\lambda_{i}=0$. We will show that $z_{\sigma(i), j} \in \mathcal{G}_{f}$ if and only if $1 \leq j<\lambda_{s}$, or $j=\lambda_{s}$ and $\sigma(i)<\max (f)$.

(i) When $1 \leq j<\lambda_{s}$, we look at the squarefree monomial $f^{\prime}:=f z_{\sigma(i), j} / z_{\sigma(s), \lambda_{s}}$, which belongs to $J$ by Corollary 2.4. Then, we can argue as in the item (i) of (A) to see that $z_{\sigma(i), j} \in J_{f}: f$.

(ii) If $j>\lambda_{s}$, we suppose for contradiction that $z_{\sigma(i), j} \in \mathcal{G}_{f}$. As in the item (iii) of (A), there exists some $f^{\prime \prime} \in G\left(J_{f}\right)$ such that $f^{\prime \prime}$ divides $f z_{\sigma(i), j}$, and $z_{\sigma(i), j}$ divides $f^{\prime \prime}$ by the minimality of $f$. Furthermore, since $f^{\prime \prime} \prec f$, we have $\operatorname{suppdeg}\left(f^{\prime \prime}\right) \leq \operatorname{suppdeg}(f)$ and $f^{\prime \prime}$ properly divides $f z_{\sigma(i), j}$. We have two subcases to consider here.

(a) If $\operatorname{suppdeg}\left(f^{\prime \prime}\right)=\operatorname{suppdeg}(f)$, there is some $i^{\prime} \neq i$ such that $\lambda_{i^{\prime}}>0$ and $f^{\prime \prime}=f z_{\sigma(i), j} / z_{\sigma\left(i^{\prime}\right), \lambda_{i^{\prime}}}$. Since $\operatorname{suppdeg}\left(f^{\prime \prime}\right)=\operatorname{suppdeg}(f)$ while $w\left(f^{\prime \prime}\right)>$ $w(f)$, this contradicts the condition $f^{\prime \prime} \prec f$.

(b) If $\operatorname{suppdeg}\left(f^{\prime \prime}\right)<\operatorname{suppdeg}(f)$, there exists some subset

$$
\mathcal{I} \subseteq\left\{k: 1 \leq k \leq s, \lambda_{k}>0\right\}
$$

such that $|\mathcal{I}| \geq 2, i \notin \mathcal{I}$ and $f^{\prime \prime}=f z_{\sigma(i), j} / \prod_{k \in \mathcal{I}} z_{\sigma(k), \lambda_{k}}$. We can fix an arbitrary $k_{0} \in \mathcal{I}$ and look at

$$
f^{\prime \prime \prime}:=f^{\prime \prime} z_{\sigma\left(k_{0}\right), \lambda_{k_{0}}} / z_{\sigma(i), j}=f / \prod_{k \in \mathcal{I} \backslash\left\{k_{0}\right\}} z_{\sigma(k), \lambda_{k}} .
$$


Since $j>\lambda_{k_{0}}$, it follows from Lemma 2.3 that $f^{\prime \prime \prime} \in J$ by considering the pair $\left(f^{\prime \prime}, f^{\prime \prime \prime}\right)$. However, $f^{\prime \prime \prime}$ divides $f$ properly. This contradicts the minimality of $f$.

Since we get contradiction in both subcases, $z_{\sigma(i), j} \notin \mathcal{G}_{f}$, as expected.

(iii) If $\sigma(i)<\max (f)$, we look at the squarefree monomial $f^{\prime}:=f z_{\sigma(i), \lambda_{s}} / z_{\max (f), \lambda_{s}}$, which belongs to $J$ by (S-3). Notice that although $\boldsymbol{\lambda}\left(f^{\prime}\right)=\boldsymbol{\lambda}(f)$, we have $f^{\prime}>_{\text {lex }} f$. Thus, $f^{\prime} \prec f$, which in turn implies $z_{\sigma(i), \lambda_{s}} \in \mathcal{G}_{f}$.

(iv) It remains to consider the situation when $\sigma(i) \geq \max (f)$. Since $\sigma(i)=$ $0<\lambda_{s}$, this amounts to saying that $\sigma(i)>\max (f)$. Now, we suppose for contradiction that $z_{\sigma(i), \lambda_{s}} \in \mathcal{G}_{f}$. Like in the case (ii), we can find some $f^{\prime \prime} \in G\left(J_{f}\right)$ such that $z_{\sigma(i), \lambda_{s}}$ divides $f^{\prime \prime}$ and $f^{\prime \prime}$ properly divides $f z_{\sigma(i), \lambda_{s}}$.

(a) If $\operatorname{suppdeg}\left(f^{\prime \prime}\right)=\operatorname{suppdeg}(f)$, there is some $i^{\prime} \neq i$ such that $\lambda_{i^{\prime}}>0$ and $f^{\prime \prime}=f z_{\sigma(i), \lambda_{s}} / z_{\sigma\left(i^{\prime}\right), \lambda_{i^{\prime}}}$. We always have $\lambda_{i^{\prime}} \leq \lambda_{s}$. If $\lambda_{i^{\prime}}<\lambda_{s}$, then $w\left(f^{\prime \prime}\right)>w(f)$, contradicting the assumption that $f^{\prime \prime} \prec f$. On the other hand, if $\lambda_{i^{\prime}}=\lambda_{s}$, then $\boldsymbol{\lambda}\left(f^{\prime \prime}\right)=\boldsymbol{\lambda}(f)$. As $\sigma(i)>\max (f) \geq \sigma\left(i^{\prime}\right)$, we have $f>_{\text {lex }} f^{\prime \prime}$. And this again contradicts the assumption that $f^{\prime \prime} \prec f$.

(b) If $\operatorname{suppdeg}\left(f^{\prime \prime}\right)<\operatorname{suppdeg}(f)$, the proof will be similar to that in item (b) of (ii), with $j$ there replaced by $\lambda_{s}$ here. Note that we can still apply Lemma 2.3 .

Since we get contradiction in both subcases, $z_{\sigma(i), \lambda_{s}} \notin \mathcal{G}_{f}$, as expected.

Thus, we have proved the Lemma 2.7.

By the definition of $\mathcal{G}_{f}$, we have $\left\langle\mathcal{G}_{f}\right\rangle \subseteq J_{f}: f$. The remaining task is to show this is actually equality.

Lemma 2.8. We have $\left\langle\mathcal{G}_{f}\right\rangle=J_{f}: f$ in general.

Proof. Suppose for contradiction that we only have $\left\langle\mathcal{G}_{f}\right\rangle \subsetneq J_{f}: f$. Then, we can find suitable $g \in G\left(J_{f}: f\right)$ such that $g \notin\left\langle\mathcal{G}_{f}\right\rangle$. We have mentioned in Remark 2.5 that this $g$ is a sparse squarefree monomial which is coprime to $f$. Thus, there exist some nonempty $\mathcal{J} \subseteq[s]:=\{1,2, \ldots, s\}$ and positive integers $\lambda_{j}^{\prime}$ for each $j \in \mathcal{J}$, such that $g=\prod_{j \in \mathcal{J}} z_{\sigma(j), \lambda_{j}^{\prime}}$. Thus, as $g \notin \mathcal{G}_{f}$ and $g$ is coprime to $f$, for each $j \in \mathcal{J}$, we have:

(i) if $\lambda_{j}>0$, then $\lambda_{j}^{\prime}>\lambda_{j}$;

(ii) if $\lambda_{j}=0$, then either $\lambda_{j}^{\prime}>\lambda_{s}$, or $\lambda_{j}^{\prime}=\lambda_{s}$ and $\sigma(j)>\max (f)$.

As $g f \in J_{f}$, we can find some $f^{\prime} \in G\left(J_{f}\right)$ dividing $g f$. Since $g$ is a minimal generator of $J_{f}: f$ while $f^{\prime}$ is a sparse squarefree monomial, we must have $f^{\prime}=g \prod_{j \in \mathcal{J}^{\prime}} z_{\sigma(j), \lambda_{j}}$ for some $\mathcal{J}^{\prime} \subset\left\{j \in[s] \backslash \mathcal{J}: \lambda_{j}>0\right\}$. Furthermore, as $f^{\prime} \prec f$, we have $\operatorname{suppdeg}\left(f^{\prime}\right) \leq$ suppdeg $(f)$. Now, we have two cases to discern.

(a) The first case is when $\operatorname{suppdeg}\left(f^{\prime}\right)<\operatorname{suppdeg}(f)$, which amounts to saying that

$$
\left|\left\{j \in \mathcal{J}: \lambda_{j}=0\right\}\right|<\left|\left\{j \notin \mathcal{J} \sqcup \mathcal{J}^{\prime}: \lambda_{j}>0\right\}\right| .
$$

Here, $\sqcup$ indicates that the union is disjoint. We may take an arbitrary subset $\mathcal{J}^{\prime \prime} \subset\left\{j \notin \mathcal{J} \sqcup \mathcal{J}^{\prime}: \lambda_{j}>0\right\}$ with a bijection $\varphi$ from $\mathcal{J}^{\prime \prime}$ to $\left\{j \in \mathcal{J}: \lambda_{j}=0\right\}$. Since $f^{\prime} \in J$, the product element

$$
\prod_{j \in \mathcal{J}: \lambda_{j}>0} z_{\sigma(j), \lambda_{j}^{\prime}} \cdot \prod_{j \in \mathcal{J}^{\prime}} z_{\sigma(j), \lambda_{j}} \cdot \prod_{j \in \mathcal{J}^{\prime \prime}} z_{\sigma(j), \lambda_{\varphi(j)}^{\prime}} \in J
$$

by the symmetry condition (S-3). In the first part of this product, each $\lambda_{j}^{\prime}>\lambda_{j}$. Meanwhile, in the third part, each $\lambda_{\varphi(j)}^{\prime} \geq \lambda_{s} \geq \lambda_{j}$. Thus, by the shiftiness 
condition (S-2), we have

$$
f^{\prime \prime}:=\prod_{j \in \mathcal{J}: \lambda_{j}>0} z_{\sigma(j), \lambda_{j}} \cdot \prod_{j \in \mathcal{J}^{\prime}} z_{\sigma(j), \lambda_{j}} \cdot \prod_{j \in \mathcal{J}^{\prime \prime}} z_{\sigma(j), \lambda_{j}} \in J .
$$

As $\operatorname{suppdeg}\left(f^{\prime \prime}\right)=\operatorname{suppdeg}\left(f^{\prime}\right)<\operatorname{suppdeg}(f), f^{\prime \prime}$ properly divides $f$, contradicting the minimality of $f$.

(b) The second case is when $\operatorname{suppdeg}\left(f^{\prime}\right)=\operatorname{suppdeg}(f)$. If we apply the notation in the previous case, then this simply means $\left\{j \in[s]: \lambda_{j}>0\right\}$ is a subset of the disjoint union $\mathcal{J} \sqcup \mathcal{J}^{\prime} \sqcup \mathcal{J}^{\prime \prime}$. It then follows from $f^{\prime} \prec f$ that $w\left(f^{\prime}\right) \leq w(f)$, which is just

$$
\sum_{\substack{j \in \mathcal{J} \\ \lambda_{j}>0}} \lambda_{j}^{\prime}+\sum_{j \in \mathcal{J}^{\prime}} \lambda_{j}+\sum_{j \in \mathcal{J}^{\prime \prime}} \lambda_{\varphi(j)}^{\prime} \leq \sum_{\substack{j \in \mathcal{J} \\ \lambda_{j}>0}} \lambda_{j}+\sum_{j \in \mathcal{J}^{\prime}} \lambda_{j}+\sum_{j \in \mathcal{J}^{\prime \prime}} \lambda_{j} .
$$

By the comparisons stated in the previous case, this is possible only when all of the following hold:

(1) there is no $j \in \mathcal{J}$ such that $\lambda_{j}>0$;

(2) for each $j \in \mathcal{J}^{\prime \prime}, \lambda_{j}=\lambda_{s}=\lambda_{\varphi(j)}^{\prime}$.

Consequently, $\boldsymbol{\lambda}\left(f^{\prime}\right)=\boldsymbol{\lambda}(f)$ and $\varphi\left(\mathcal{J}^{\prime \prime}\right)=\left\{j \in \mathcal{J}: \lambda_{j}=0\right\}=\mathcal{J}$. Furthermore, since

$$
\mathcal{J}^{\prime} \sqcup \mathcal{J}^{\prime \prime} \subseteq\left\{j: \lambda_{j}>0\right\} \subseteq \mathcal{J} \sqcup \mathcal{J}^{\prime} \sqcup \mathcal{J}^{\prime \prime},
$$

it follows again from the above item (1) that

$$
\left\{j \in[s]: \lambda_{j}>0\right\}=\mathcal{J}^{\prime} \sqcup \mathcal{J}^{\prime \prime} .
$$

And as $\mathcal{J}=\varphi\left(\mathcal{J}^{\prime \prime}\right)$, we have $\lambda_{j}^{\prime}=\lambda_{s}$ for each $j \in \mathcal{J}$, by the above item (2). Hence, we can observe that

- for each $j \in[s] \backslash \mathcal{J}^{\prime}$ with $\lambda_{j} \neq \lambda_{j}^{\prime}, j$ belongs to $\mathcal{J}^{\prime \prime}$ or $\mathcal{J}$, which means that $\left(\lambda_{j}, \lambda_{j}^{\prime}\right)$ equals either $\left(\lambda_{s}, 0\right)$ or $\left(0, \lambda_{s}\right)$ correspondingly;

- for each $j \in[s] \backslash \mathcal{J}^{\prime}$ with $\left(\lambda_{j}, \lambda_{j}^{\prime}\right)=\left(0, \lambda_{s}\right)$, one has $j \in \mathcal{J}$ and $\sigma(j)>\max (f)$ by the property of $g$.

Since $\mathcal{J} \neq \varnothing$, it follows that $f>_{\text {lex }} f^{\prime}$, contradicting to the fact $f^{\prime} \in G\left(J_{f}\right)$.

Therefore, we have shown the claimed equality $\left\langle\mathcal{G}_{f}\right\rangle=J_{f}: f$.

Now, $J_{f}: f$ is generated by the variables in $\mathcal{G}_{f}$. In particular, it is linear. Since this holds for each $f \in G(J), J$ has linear quotients, as expected.

2.2. Betti Numbers. In the following, $J$ is a sparse symmetric shifted ideal. Like [3, Corollary 5.7], we want to calculate the graded Betti number of $J$. The main arguments for that purpose follow closely from those in [3, Section 5]. Some necessary details are included here, mainly to keep the exposition self-contained.

Let $\Lambda(J):=\{\boldsymbol{\lambda}(f): f \in G(J)\}$ be the set of partitions of the minimal monomial generators of $J$. Without loss of generality, we may assume that $\Lambda(J)=\left\{\boldsymbol{\lambda}^{(1)}, \ldots, \boldsymbol{\lambda}^{(t)}\right\}$ with $\boldsymbol{\lambda}^{(1)} \prec \cdots \prec \boldsymbol{\lambda}^{(t)}$. Here, $\prec$ is the total order we introduced in the proof of Theorem 2.6. For each $k$ with $1 \leq k \leq t$, let $J_{\leq k} \subseteq J$ be the sparse symmetric shifted ideal with $\Lambda\left(J_{\leq k}\right)=\left\{\boldsymbol{\lambda}^{(1)}, \ldots, \boldsymbol{\lambda}^{(k)}\right\}$. Thus, we have a short exact sequence

$$
0 \rightarrow J_{\leq k-1} \rightarrow J_{\leq k} \rightarrow J_{\leq k} / J_{\leq k-1} \rightarrow 0 .
$$

Notice that the quotient in the above sequence can be better recognized as

$$
J_{\leq k} / J_{\leq k-1} \cong\left\langle\sigma\left(\boldsymbol{z}^{\boldsymbol{\lambda}^{(k)}}\right): \sigma \in \mathfrak{S}_{s}\right\rangle /\left(\left\langle\sigma\left(\boldsymbol{z}^{\boldsymbol{\lambda}^{(k)}}\right): \sigma \in \mathfrak{S}_{s}\right\rangle \cap J_{\leq k-1}\right) .
$$


Lemma 2.9. Assume that $\boldsymbol{\lambda}^{(k)}=\left(\lambda_{1}, \ldots, \lambda_{s}\right)$. Then, the intersection ideal $K:=$ $\left\langle\mathfrak{S}_{s} \boldsymbol{z}^{\boldsymbol{\lambda}^{(k)}}\right\rangle \cap J_{\leq k-1}$ is the sum of the ideal

$$
K_{1}:=\left\langle\sigma\left(\boldsymbol{z}^{\boldsymbol{\lambda}^{(k)}} z_{i, j}\right): \sigma \in \mathfrak{S}_{s}, \lambda_{i}>0 \text { and } 1 \leq j<\lambda_{i}\right\rangle
$$

with the ideal

$$
K_{2}:=\left\langle\sigma\left(\boldsymbol{z}^{\boldsymbol{\lambda}^{(k)}} z_{i, j}\right): \sigma \in \mathfrak{S}_{s}, \lambda_{i}=0 \text { and } 1 \leq j<\lambda_{s}\right\rangle .
$$

Proof. We first show the containment " $K \supseteq K_{1}+K_{2}$ ". Let $f=z^{\mu}$ be the first monomial in $G\left(J_{\leq k}\right) \backslash G\left(J_{\leq k-1}\right)$ with respect to the order $\prec$ on $G(J)$. Then, for some $\tau \in \mathfrak{S}_{s}$, we have $f=\tau\left(\boldsymbol{z}^{\boldsymbol{\lambda}^{(k)}}\right)=\prod_{i} z_{\tau(i), \lambda_{i}}$, and $J_{f}: f=J_{\leq k-1}: f=\left\langle\mathcal{G}_{f}\right\rangle$. Notice that in this case,

$$
\mathcal{G}_{f}=\left\{z_{\tau(i), j}: \lambda_{i}>0 \text { and } 1 \leq j<\lambda_{i}\right\} \cup\left\{z_{\tau(i), j}: \lambda_{i}=0 \text { and } 1 \leq j<\lambda_{s}\right\} .
$$

Clearly, the lexicographic order is used here to make the third type of sets in Lemma 2.7 empty, via the item (B)|(iii) in its proof. Now, for each $z_{\tau(i), \lambda_{j}} \in \mathcal{G}_{f}$, one has $f z_{\tau(i), \lambda_{j}}=$ $\tau\left(\boldsymbol{z}^{\boldsymbol{\lambda}^{(k)}} z_{i, j}\right) \in J_{\leq k-1}$. And the containment " $\supseteq$ " follows from the $\mathfrak{S}_{s}$ actions.

Next, we show the opposite containment " $K \subseteq K_{1}+K_{2}$ ". For this purpose, we take arbitrary squarefree monomial $g$ in $\left\langle\mathfrak{S}_{s} \boldsymbol{z}^{\boldsymbol{\lambda}^{(k)}}\right\rangle \cap J_{\leq k-1}$. We will also utilize the generator $f=\tau\left(\boldsymbol{z}^{\boldsymbol{\lambda}^{(k)}}\right)$ in the previous paragraph. Now, $\tau^{\prime}(g) \in\langle f\rangle$ for some $\tau^{\prime} \in \mathfrak{S}_{s}$. Thus, $\tau^{\prime}(g)=g_{1} f$ for some squarefree monomial $g_{1}$. Since $\tau^{\prime}(g) \in J_{\leq k-1}, g_{1} \in J_{\leq k-1}: f=\left\langle\mathcal{G}_{f}\right\rangle$, which was described in the above equation (1). Now the containment " $\subseteq$ " follows from the $\mathfrak{S}_{s}$ actions.

In the following, for each partition $\boldsymbol{\lambda}=\left(\lambda_{1}, \ldots, \lambda_{s}\right)$, we will write $N^{\boldsymbol{\lambda}}$ for the quotient module

$$
\frac{\left\langle\sigma\left(\boldsymbol{z}^{\boldsymbol{\lambda}}\right): \sigma \in \mathfrak{S}_{s}\right\rangle}{\left\langle\sigma\left(\boldsymbol{z}^{\boldsymbol{\lambda}} z_{i, j}\right): \lambda_{i}>0,1 \leq j<\lambda_{i}, \sigma \in \mathfrak{S}_{s}\right\rangle+\left\langle\sigma\left(\boldsymbol{z}^{\boldsymbol{\lambda}} z_{i, j}\right): \lambda_{i}=0,1 \leq j<\lambda_{s}, \sigma \in \mathfrak{S}_{s}\right\rangle} .
$$

Corollary 2.10. One has $J_{\leq k} / J_{\leq k-1} \cong N^{\boldsymbol{\lambda}^{(k)}}$.

Next, we want to describe the $\mathbb{K}$-linear structure of $N^{\boldsymbol{\lambda}}$. Let $N_{\text {num }}^{\boldsymbol{\lambda}}$ and $N_{\text {denom }}^{\boldsymbol{\lambda}}$ be the squarefree monomial ideals in the numerator and denominator of $N^{\boldsymbol{\lambda}}$ respectively. Since $N^{\boldsymbol{\lambda}}$ is a squarefree module, it suffices to take an arbitrary squarefree monomial $g \in N_{\text {num }}^{\lambda}$ and ask when $g \notin N_{\text {denom }}^{\lambda}$. For each $i \in[s]$, if some $z_{i, j}$ divides $g$, let $\mu_{i}=$ $\min \left\{j: z_{i, j}\right.$ divides $\left.g\right\}$. If no such $z_{i, j}$ exists for this chosen $i$, let $\mu_{i}=0$. Thus, we have a tuple $\boldsymbol{\mu}=\left(\mu_{1}, \ldots, \mu_{s}\right) \in \mathbb{Z}_{\geq 0}^{s}$ for this squarefree monomial $g$. Additionally, we may assume that the partition associated with $\boldsymbol{z}^{\mu}$ is $\boldsymbol{\lambda}^{\prime}=\left(\lambda_{1}^{\prime}, \ldots, \lambda_{s}^{\prime}\right)$. Suppose that $\theta=\operatorname{suppdeg}(\boldsymbol{\lambda})$ and $\theta^{\prime}=\operatorname{suppdeg}\left(\boldsymbol{\lambda}^{\prime}\right)$. Thus, $\lambda_{s-\theta}=0<\lambda_{s-\theta+1}$ and similarly $\lambda_{s-\theta^{\prime}}^{\prime}=0<\lambda_{s-\theta^{\prime}+1}^{\prime}$

Lemma 2.11. With the notations as above, the squarefree monomial $g$ belongs to $N_{\text {num }}^{\lambda} \backslash$ $N_{\text {denom }}^{\lambda}$ if and only if

$$
\theta \leq \theta^{\prime} \quad \text { and } \quad \lambda_{s-\theta+k}=\lambda_{s-\theta^{\prime}+k}^{\prime} \text { for } k=1,2, \ldots, \theta .
$$

Proof. Suppose that the squarefree monomial $g$ belongs to $N_{\text {num }}^{\boldsymbol{\lambda}} \backslash N_{\text {denom }}^{\boldsymbol{\lambda}}$. By symmetry, we may assume that $\boldsymbol{z}^{\boldsymbol{\lambda}}$ divides $g$. We observe the following two facts.

(a) Firstly, whenever $\lambda_{i}>0, z_{i, \lambda_{i}}$ divides $g$. Therefore, $\mu_{i} \leq \lambda_{i}$. However, when $\mu_{i}<\lambda_{i}$ for one such $i$, then $g \in N_{\text {denom }}^{\lambda}$ as well. Thus, we have precisely $\mu_{i}=\lambda_{i}$ whenever $\lambda_{i}>0$.

(b) Secondly, for each $i$ with $\lambda_{i}=0$, if $1 \leq \mu_{i}<\lambda_{s}$, then $g \in N_{\text {denom }}^{\lambda}$ as well. Thus either $\mu_{i}=0$ or $\mu_{i} \geq \lambda_{s}$. 
From these observations, we see immediately that condition (2) holds.

Conversely, suppose that condition (2) is satisfied. It is clear that $\boldsymbol{z}^{\boldsymbol{\lambda}}$ divides $\tau\left(\boldsymbol{z}^{\boldsymbol{\lambda}^{\prime}}\right)$ for some permutation $\tau \in \mathfrak{S}_{s}$. Meanwhile, $\tau^{\prime}\left(\boldsymbol{z}^{\boldsymbol{\lambda}^{\prime}}\right)=\boldsymbol{z}^{\mu}$ divides $g$ for some permutation $\tau^{\prime} \in \mathfrak{S}_{s}$. Thus, $g \in\left\langle\sigma\left(\boldsymbol{z}^{\boldsymbol{\lambda}}\right): \sigma \in \mathfrak{S}_{s}\right\rangle=N_{\text {num }}^{\boldsymbol{\lambda}}$. As for testing whether $g \in N_{\text {denom }}^{\boldsymbol{\lambda}}$, we are reduced to the following two cases by symmetry.

(i) Suppose that $g$ is divisible by $\boldsymbol{z}^{\boldsymbol{\lambda}} z_{i_{0}, j}$ for some $1 \leq j<\lambda_{i_{0}}$ with $\lambda_{i_{0}}>0$. Then, whenever $\lambda_{i}>0$, we have $\mu_{i} \leq \lambda_{i}$. Furthermore, $\mu_{i_{0}}<\lambda_{i_{0}}$. This will break the assumptions in (2).

(ii) Suppose that $g$ is divisible by $\boldsymbol{z}^{\boldsymbol{\lambda}} z_{i_{0}, j}$ for some $1 \leq j<\lambda_{s}$ with $\lambda_{i_{0}}=0$. Then, whenever $\lambda_{i}>0$, we have $\mu_{i} \leq \lambda_{i}$. Furthermore, $1 \leq \mu_{i_{0}}<\lambda_{s}$. This still breaks the assumptions in (2).

Thus, $g \in N_{\text {num }}^{\boldsymbol{\lambda}} \backslash N_{\text {denom }}^{\boldsymbol{\lambda}}$.

It is time to introduce a series of new notations.

Notation 2.12. Given any partition $\boldsymbol{\lambda}=\left(\lambda_{1}, \ldots, \lambda_{s}\right)$, we may assume that

$$
\boldsymbol{\lambda}=\left(0, \ldots, 0, \lambda_{k_{1}}, \lambda_{k_{1}+1}, \ldots, \lambda_{k_{2}}, \lambda_{s}, \ldots, \lambda_{s}\right)
$$

where $\lambda_{k_{1}} \neq 0$ and $\lambda_{k_{2}}<\lambda_{s}$.

- Let $\pi_{\boldsymbol{\lambda}}:=\left(\lambda_{k_{1}}, \lambda_{k_{1}+1}, \ldots, \lambda_{k_{2}}\right)$ be the non-zero and non-maximal piece of $\boldsymbol{\lambda}$. Write $p(\boldsymbol{\lambda}):=k_{2}-k_{1}+1=\left|\left\{j: 1 \leq \lambda_{j}<\lambda_{s}\right\}\right|$ for the length of this piece and $r(\boldsymbol{\lambda}):=$ $\left|\left\{j: \lambda_{j}=\lambda_{s}\right\}\right|$ for the length of the remaining maximal piece.

- For any $p(\boldsymbol{\lambda})$-subset $A=\left\{i_{1}, i_{2}, \ldots, i_{p(\boldsymbol{\lambda})}\right\} \subseteq[s]$ with $i_{1}<i_{2}<\cdots<i_{p(\boldsymbol{\lambda})}$, and any positive integer $a$, let $Z_{A, a}:=\mathbb{K}\left[z_{i, j} \in Z: i \in A\right.$ and $\left.j \geq a\right]$. When $a=1$, we may write this subring simply as $Z_{A}$ and write $\boldsymbol{z}_{A}^{\pi_{\boldsymbol{\lambda}}}:=\prod_{j=1}^{p(\boldsymbol{\lambda})} z_{i_{j}, \lambda_{k_{1}+j-1}} \in Z_{A}$.

- With respect to the subset $A$ above, we write $A^{\complement}:=[s] \backslash A$ for the complement set, and similarly introduce $Z_{A^{\complement}, a}:=\mathbb{K}\left[z_{i, j} \in Z: i \in A^{\complement}\right.$ and $\left.j \geq a\right]$ for the positive integer $a$.

- For the complement set $A^{\complement}$, we will write $V_{A^{\complement}, \boldsymbol{\lambda}}$ for the monomial ideal in $Z_{A^{\complement}, \lambda_{s}}$ generated by the sparse squarefree monomials of the form $z_{t_{1}, \lambda_{s}} z_{t_{2}, \lambda_{s}} \cdots z_{t_{r(\boldsymbol{\lambda})}, \lambda_{s}}$ with $t_{1}, t_{2}, \ldots, t_{r(\boldsymbol{\lambda})}$ distinct in $A^{\complement}$.

Notice that we have a natural group action of $\mathfrak{S}_{A}$ on $Z_{A, a}$, which can be derived from the action of $\mathfrak{S}_{s}$ on $Z_{A, a}$.

Remark 2.13. Fix a sparse squarefree monomial $m=\prod_{j=1}^{p(\boldsymbol{\lambda})} z_{i_{j}, \mu_{j}} \in \mathfrak{S}_{A} \boldsymbol{z}_{A}^{\pi_{\boldsymbol{\lambda}}}$ with $\boldsymbol{\mu}=$ $\left(\mu_{1}, \ldots, \mu_{p(\boldsymbol{\lambda})}\right)$. Surely we have $m m^{\prime} \in \mathfrak{S}_{s} \boldsymbol{z}^{\boldsymbol{\lambda}}$ for each sparse monomial $m^{\prime} \in G\left(V_{A^{\complement}, \boldsymbol{\lambda}}\right)$. It is not difficult to deduce from Lemma 2.11 that the annihilating ideal of the image of $m m^{\prime}$ is given by

$$
\left\langle z_{i, j} \text { : either } i \in A \text { and } 1 \leq j<\mu_{i} \text {, or } i \notin A \text { and } 1 \leq j<\lambda_{s}\right\rangle,
$$

which is independent of the choice of $m^{\prime}$.

Notation 2.14. Inspired by the description of (3), we introduce the following subrings, submodule, and ideals.

- For any given $m=\prod_{j=1}^{p(\boldsymbol{\lambda})} z_{i_{j}, \mu_{j}} \in \mathfrak{S}_{A} \boldsymbol{z}_{A}^{\pi_{\boldsymbol{\lambda}}}$ with $\boldsymbol{\mu}=\left(\mu_{1}, \ldots, \mu_{p(\boldsymbol{\lambda})}\right)$, let

$$
Z_{m}^{\Delta}:=\mathbb{K}\left[z_{i, j} \text { : either } i \in A \text { and } 1 \leq j<\mu_{i} \text {, or } i \notin A \text { and } 1 \leq j<\lambda_{s}\right] \text {, }
$$

and write $\mathfrak{m}_{Z_{m}}$ for its graded maximal ideal. Similarly, let

$$
Z_{m}^{\nabla}:=\mathbb{K}\left[z_{i, j}: \mu_{i} \leq j \leq b \text { and } i \in A\right] \subset Z_{A} .
$$


Roughly speaking, the variables in $Z_{m}^{\Delta}$ are in the upper half of the canonical matrix $\boldsymbol{Z}$ consider in Remark 2.2, while the variables in $Z_{m}^{\nabla}$ are in the lower half of the matrix.

- For this given $m$, we write $N_{A, m}^{\lambda}$ for the submodule generated by the image of $m V_{A^{\mathrm{C}}, \boldsymbol{\lambda}}$ in $N^{\boldsymbol{\lambda}}$.

- Additionally, we write $V_{A^{\mathrm{C}}, \boldsymbol{\lambda}}^{*}$ for the squarefree Veronese ideal of degree $r(\boldsymbol{\lambda})$ in $\mathbb{K}\left[z_{i, \lambda_{s}}: i \in A^{\complement}\right]$, which is generated by the squarefree monomials of degree $r(\boldsymbol{\lambda})$ in this ring.

Remark 2.15. It is clear that

$$
V_{A^{\complement}, \boldsymbol{\lambda}}=V_{A^{\mathrm{C}}, \boldsymbol{\lambda}}^{*} Z_{A^{\mathrm{C}}, \lambda_{s}} \cong V_{A^{\mathrm{C}}, \boldsymbol{\lambda}}^{*} \otimes_{\mathbb{K}} Z_{A^{\complement}, \lambda_{s}+1}
$$

is an extended ideal from $V_{A^{\mathrm{C}}, \boldsymbol{\lambda}}^{*}$. Meanwhile, we also have that multigraded isomorphism

$$
N_{A, m}^{\lambda} \cong Z_{m}^{\Delta} / \mathfrak{m}_{Z_{m}^{\Delta}} \otimes_{\mathbb{K}} m Z_{m}^{\nabla} \otimes_{\mathbb{K}} V_{A^{\complement}, \boldsymbol{\lambda}}^{*} \otimes_{\mathbb{K}} Z_{A^{\complement}, \lambda_{s}+1} .
$$

Thus, we can deduce from Lemma 2.11 and the multidegree information in (5) the following conclusion.

Corollary 2.16. We have the following decomposition as multigraded $Z$-modules:

$$
N^{\boldsymbol{\lambda}}=\bigoplus_{\substack{A \subset[s] \\|A|=p(\boldsymbol{\lambda})}} \bigoplus_{m \in \mathfrak{S}_{A} \boldsymbol{z}_{A}^{\pi_{\boldsymbol{\lambda}}}} N_{A, m}^{\boldsymbol{\lambda}} .
$$

Notice that the monomial $m$ sits only in $Z_{A}$ and has degree $\operatorname{deg}\left(\boldsymbol{z}_{A}^{\pi_{\boldsymbol{\lambda}}}\right)=p(\boldsymbol{\lambda})$. Since $V_{A^{\mathrm{C}}, \boldsymbol{\lambda}}^{*}$ is the squarefree Veronese ideal of degree $r(\boldsymbol{\lambda})$ in a suitable ring. It follows from (44) that $V_{A^{\mathrm{C}}, \boldsymbol{\lambda}}$ in $Z_{A^{\mathrm{C}}, \lambda_{s}}$ has a linear resolution with regularity $r(\boldsymbol{\lambda})$. Consequently, both $N_{A, m}^{\boldsymbol{\lambda}}$ and $N^{\boldsymbol{\lambda}}$ have linear resolutions with regularity $p(\boldsymbol{\lambda})+r(\boldsymbol{\lambda})=\operatorname{suppdeg}(\boldsymbol{\lambda})$.

Now, we can state the second main result of this section.

Theorem 2.17. If $J$ is a sparse symmetric shifted ideal, then as $\mathbb{K}\left[\mathfrak{S}_{s}\right]$-modules, we have

$$
\operatorname{Tor}_{i}(J, \mathbb{K})_{i+d} \cong \bigoplus_{\substack{\boldsymbol{\lambda} \in \Lambda(J) \\ \operatorname{suppdeg}(\boldsymbol{\lambda})=d}} \operatorname{Tor}_{i}\left(N^{\boldsymbol{\lambda}}, \mathbb{K}\right)
$$

Its proof is literally the same as the argument for [3, Theorem 5.5]. There is no need repeating it here.

It remains to give a closed formula of the graded Betti numbers of $J$ using the information from $\Lambda(J)$. To achieve this, consider the case when $\operatorname{suppdeg}(\boldsymbol{\lambda})=d, A \subset[s]$ with $|A|=p(\boldsymbol{\lambda})$, and $m=\prod_{j=1}^{p(\boldsymbol{\lambda})} z_{i_{j}, \mu_{j}} \in \mathfrak{S}_{A} \boldsymbol{z}_{A}^{\pi_{\boldsymbol{\lambda}}}$. Notice that the dimension of $Z_{m}^{\Delta}$ is precisely

$$
\begin{aligned}
p^{\Delta}(\boldsymbol{\lambda}) & :=\left|\pi_{\boldsymbol{\lambda}}\right|-p(\boldsymbol{\lambda})+\left(\lambda_{s}-1\right)(s-p(\boldsymbol{\lambda})) \\
& =|\boldsymbol{\lambda}|+s\left(\lambda_{s}-1\right)-\lambda_{s} \operatorname{suppdeg}(\boldsymbol{\lambda}) .
\end{aligned}
$$

Thus, we can deduce from (5) that

$$
\begin{aligned}
\beta_{i, i+d}\left(N_{A, m}^{\boldsymbol{\lambda}}\right) & =\beta_{i}\left(Z_{m}^{\Delta} / \mathfrak{m}_{Z_{m}^{\Delta}} \otimes_{\mathbb{K}} Z_{m}^{\nabla} \otimes_{\mathbb{K}} V_{A^{\complement}, \boldsymbol{\lambda}}^{*} \otimes_{\mathbb{K}} Z_{A^{\complement}, \lambda_{s}+1}\right) \\
& =\sum_{k+\ell=i} \beta_{\ell}\left(Z_{m}^{\Delta} / \mathfrak{m}_{Z_{m}^{\Delta}}\right) \beta_{k}\left(V_{A^{\complement}, \boldsymbol{\lambda}}^{*}\right) \\
& =\sum_{k+\ell=i}\left(\begin{array}{c}
p^{\Delta}(\boldsymbol{\lambda}) \\
\ell
\end{array}\right)\left(\begin{array}{c}
s-p(\boldsymbol{\lambda}) \\
r(\boldsymbol{\lambda})+k
\end{array}\right)\left(\begin{array}{c}
r(\boldsymbol{\lambda})+k-1 \\
k
\end{array}\right) ;
\end{aligned}
$$


see also [9, Theorem 2.1].

Meanwhile, we will define the term type $\left(\pi_{\boldsymbol{\lambda}}\right):=\left(t_{1}, \ldots, t_{\lambda_{s-1}}\right)$ where $t_{k}=\left|\left\{j: \lambda_{j}=k\right\}\right|$ for $1 \leq k \leq \lambda_{s-1}$. And it is clear that $\left|\mathfrak{S}_{A} \boldsymbol{z}_{A}^{\pi_{\boldsymbol{\lambda}}}\right|=\frac{p(\boldsymbol{\lambda}) !}{\operatorname{type}\left(\pi_{\boldsymbol{\lambda}}\right) !}$, where type $\left(\pi_{\boldsymbol{\lambda}}\right) !:=t_{1} ! t_{2} ! \cdots t_{\lambda_{s-1}}$ !. Consequently, we have the following closed formula for Betti numbers.

Corollary 2.18. If $J$ is a sparse symmetric shifted ideal, then $\beta_{i, i+d}(J)$ is given by

$$
\sum_{\substack{\boldsymbol{\lambda} \in \Lambda(J) \\
\operatorname{suppdeg}(\boldsymbol{\lambda})=d}}\left(\sum_{k+\ell=i} \frac{p(\boldsymbol{\lambda}) !}{\operatorname{type}\left(\pi_{\boldsymbol{\lambda}}\right) !}\left(\begin{array}{c}
p^{\Delta}(\boldsymbol{\lambda}) \\
\ell
\end{array}\right)\left(\begin{array}{c}
s \\
p(\boldsymbol{\lambda})
\end{array}\right)\left(\begin{array}{c}
s-p(\boldsymbol{\lambda}) \\
r(\boldsymbol{\lambda})+k
\end{array}\right)\left(\begin{array}{c}
r(\boldsymbol{\lambda})+k-1 \\
k
\end{array}\right)\right) .
$$

A quick example using this formula is given in Example 3.4 .

\section{Sequentially Cohen-Macaulay property of Uniform $a$-Fold Product IDEALS}

In this section, we will show that the symbolic powers of the uniform $a$-fold product ideal are sequentially Cohen-Macaulay under some generic conditions. From now on, $\mathcal{F}_{\boldsymbol{z}}$ will be the list of variables $\left\{z_{1}, \ldots, z_{s}\right\}$ in the polynomial $\operatorname{ring} T=\mathbb{K}\left[z_{1}, \ldots, z_{s}\right]$. And the field $\mathbb{K}$ is infinite.

\subsection{Standard monomial case.}

Proposition 3.1. Suppose that $I=\bigcap_{k=1}^{t} I_{c_{k}, \mathcal{F}_{z}}^{\left(m_{k}\right)} \subset T$ is an intersection of symbolic powers of monomial star configuration ideals. Then, I is sequentially Cohen-Macaulay.

Proof. To show that this ideal is sequentially Cohen-Macaulay, we will apply the polarization technique. And here is a list of facts that we will utilize. They are in Propositions $2.3,2.5$ and 4.11 of [8] respectively.

- We have $\operatorname{Pol}\left(I_{1} \cap I_{2}\right)=\operatorname{Pol}\left(I_{1}\right) \cap \operatorname{Pol}\left(I_{2}\right)$.

- The polarization of $\left\langle z_{i_{1}}, \ldots, z_{i_{r}}\right\rangle^{m}$ has the following irredundant irreducible primary decomposition:

$$
\operatorname{Pol}\left(\left\langle z_{i_{1}}, \ldots, z_{i_{r}}\right\rangle^{m}\right)=\bigcap_{\substack{1 \leq e_{j} \leq m, \sum_{j} e_{j} \leq m+r-1}}\left\langle z_{i_{1}, e_{1}}, \ldots, z_{i_{r}, e_{r}}\right\rangle .
$$

- The ideal $I$ is sequentially Cohen-Macaulay if and only if $\operatorname{Pol}(I)$ is so.

Now, we will apply the polarization to the ideals in the intersection

$$
I=\bigcap_{k=1}^{t} I_{c_{k}, \mathcal{F}_{\boldsymbol{z}}}^{\left(m_{k}\right)}=\bigcap_{k=1}^{t} \bigcap_{1 \leq i_{1}<\cdots<i_{c_{k}} \leq s}\left\langle z_{i_{1}}, \ldots, z_{i_{c_{k}}}\right\rangle^{m_{k}} .
$$

Notice that the squarefree monomial ideal $\operatorname{Pol}(I)$ is sequentially Cohen-Macaulay, if and only if the Alexander dual ideal $\operatorname{Pol}(I)^{\vee}$ is componentwise linear, by [15, Theorem 8.2.20]. And now it suffices to show that $\operatorname{Pol}(I)^{\vee}$ has linear quotients, by [15, Theorem 8.2.15].

Observe that $\operatorname{Pol}(I)^{\vee}$ is generated by monomials of the form $\prod_{j=1}^{c_{k}} z_{i_{j}, e_{j}}$, where $1 \leq k \leq$ $t, 1 \leq i_{1}<\cdots<i_{c_{k}} \leq s, 1 \leq e_{j} \leq m_{k}$ and $\sum_{j} e_{j} \leq m_{k}+c_{k}-1$. It is clear that $\operatorname{Pol}(I)^{\vee}$ is sparse symmetric shifted. Thus, by Theorem 2.6, this ideal has linear quotients, as expected. And this completes our proof for Proposition 3.3 .

In the remaining of this paper, we will stay with the following setup. 
Setting 3.2. Let $a, b$ be positive integers such that $(b-1) s+1<a \leq b s$. And we introduce the positive integers

$$
h:=b s-a+1, \quad c_{0}:=s-\left\lfloor\frac{a-1}{b}\right\rfloor \quad \text { and } \quad \mu_{a}^{0}:=a-b\left(s-c_{0}\right) .
$$

Notice that if $a \leq(b-1) s+1$, then $I_{a}\left(\mathcal{F}_{z}^{b}\right)$ is not saturated by [17, Proposition 4.3]. And under Setting 3.2, the big height of $I_{a}\left(\mathcal{F}_{z}^{b}\right)$ is precisely $h$ by [17, Remark 4.5]. The main result of this subsection is the following.

Proposition 3.3. Assume the notations in Setting 3.2. Then, the symbolic powers of $I_{a}\left(\mathcal{F}_{\boldsymbol{z}}^{b}\right)=I_{a}\left(z_{1}^{b} \cdots z_{s}^{b}\right)$ are all sequentially Cohen-Macaulay.

Proof. It follows from [17, Theorem 4.6] that the symbolic power

$$
I_{a}\left(\mathcal{F}_{\boldsymbol{z}}^{b}\right)^{(m)}=\bigcap_{c=c_{0}}^{h} I_{c, \mathcal{F}_{\boldsymbol{z}}}^{\left(m\left(\mu_{a}^{0}+b\left(c-c_{0}\right)\right)\right)}
$$

for each positive integer $m$. Now, it remains to apply Proposition 3.1 .

Example 3.4. Here, we consider a small example where $I=I_{6}\left(z_{1}^{2} z_{2}^{2} z_{3}^{2} z_{4}^{2}\right)$ in $\mathbb{K}\left[z_{1}, z_{2}, z_{3}, z_{4}\right]$. Now, the squarefree monomial ideal $J=\operatorname{Pol}(I)^{\vee}$ in $\mathbb{K}\left[z_{1,1}, z_{2,1}, z_{3,1}, z_{4,1}, z_{1,2}, z_{2,2}, z_{3,2}, z_{4,2}\right]$ is sparse symmetric shifted, and has 22 minimal monomial generators. We can arrange these monomials using the total order $\prec$ in the proof of Theorem 2.6, and denote them by $u_{1}, \ldots, u_{22}$. Table 1 shows all the linear quotients $J_{u_{i}}: u_{i}$, as described by Lemma 2.7 . After applying either the well-known formula [15, Corollary 8.2.2] or Corollary 2.18, we can obtain the following Betti diagram for $J$ :

$\begin{array}{rrrrrrr} & 0 & 1 & 2 & 3 & 4 & 5 \\ \text { total : } & 22 & 75 & 115 & 94 & 40 & 7 \\ 1: & 18 & 56 & 79 & 60 & 24 & 4 \\ 2: & 4 & 19 & 36 & 34 & 16 & 3\end{array}$

Both the linear quotients and the Betti diagram agree with the outputs of Macaulay2 [13.

TABLE 1. Linear quotients of a sparse symmetric shifted ideal

\begin{tabular}{cccc}
\hline$i$ & $u_{i}$ & $J_{u_{i}}: u_{i}$ & $\max \left(u_{i}\right)$ \\
\hline 1 & $z_{1,1} z_{2,1}$ & - & 2 \\
2 & $z_{1,1} z_{3,1}$ & $\left\langle z_{2,1}\right\rangle$ & 3 \\
3 & $z_{1,1} z_{4,1}$ & $\left\langle z_{3,1}, z_{2,1}\right\rangle$ & 4 \\
4 & $z_{2,1} z_{3,1}$ & $\left\langle z_{1,1}\right\rangle$ & 3 \\
5 & $z_{2,1} z_{4,1}$ & $\left\langle z_{3,1}, z_{1,1}\right\rangle$ & 4 \\
6 & $z_{3,1} z_{4,1}$ & $\left\langle z_{2,1}, z_{1,1}\right\rangle$ & 4 \\
7 & $z_{1,1} z_{2,2}$ & $\left\langle z_{4,1}, z_{3,1}, z_{2,1}\right\rangle$ & 2 \\
8 & $z_{1,1} z_{3,2}$ & $\left\langle z_{2,2}, z_{4,1}, z_{3,1}, z_{2,1}\right\rangle$ & 3 \\
9 & $z_{1,1} z_{4,2}$ & $\left\langle z_{3,2}, z_{2,2}, z_{4,1}, z_{3,1}, z_{2,1}\right\rangle$ & 4 \\
10 & $z_{2,1} z_{1,2}$ & $\left\langle z_{4,1}, z_{3,1}, z_{1,1}\right\rangle$ & 1 \\
11 & $z_{2,1} z_{3,2}$ & $\left\langle z_{1,2}, z_{4,1}, z_{3,1}, z_{1,1}\right\rangle$ & 3 \\
\hline
\end{tabular}

\begin{tabular}{cccc}
\hline$i$ & $u_{i}$ & $J_{u_{i}}: u_{i}$ & $\max \left(u_{i}\right)$ \\
\hline 12 & $z_{2,1} z_{4,2}$ & $\left\langle z_{3,2}, z_{1,2}, z_{4,1}, z_{3,1}, z_{1,1}\right\rangle$ & 4 \\
13 & $z_{3,1} z_{1,2}$ & $\left\langle z_{4,1}, z_{2,1}, z_{1,1}\right\rangle$ & 1 \\
14 & $z_{3,1} z_{2,2}$ & $\left\langle z_{1,2}, z_{4,1}, z_{2,1}, z_{1,1}\right\rangle$ & 2 \\
15 & $z_{3,1} z_{4,2}$ & $\left\langle z_{2,2}, z_{1,2}, z_{4,1}, z_{2,1}, z_{1,1}\right\rangle$ & 4 \\
16 & $z_{4,1} z_{1,2}$ & $\left\langle z_{3,1}, z_{2,1}, z_{1,1}\right\rangle$ & 1 \\
17 & $z_{4,1} z_{2,2}$ & $\left\langle z_{1,2}, z_{3,1}, z_{2,1}, z_{1,1}\right\rangle$ & 2 \\
18 & $z_{4,1} z_{3,2}$ & $\left\langle z_{2,2}, z_{1,2}, z_{3,1}, z_{2,1}, z_{1,1}\right\rangle$ & 3 \\
19 & $z_{1,2} z_{2,2} z_{3,2}$ & $\left\langle z_{4,1}, z_{3,1}, z_{2,1}, z_{1,1}\right\rangle$ & 3 \\
20 & $z_{1,2} z_{2,2} z_{4,2}$ & $\left\langle z_{3,2}, z_{4,1}, z_{3,1}, z_{2,1}, z_{1,1}\right\rangle$ & 4 \\
21 & $z_{1,2} z_{3,2} z_{4,2}$ & $\left\langle z_{2,2}, z_{4,1}, z_{3,1}, z_{2,1}, z_{1,1}\right\rangle$ & 4 \\
22 & $z_{2,2} z_{3,2} z_{4,2}$ & $\left\langle z_{1,2}, z_{4,1}, z_{3,1}, z_{2,1}, z_{1,1}\right\rangle$ & 4 \\
\hline
\end{tabular}

3.2. General case. Notice that in the standard monomial case, the big height of $I_{a}\left(\mathcal{F}_{\boldsymbol{z}}^{b}\right)$ is $h=b s-a+1$ by [17, Remark 4.5]. Thus, for the general case when $I=I_{a}\left(\mathcal{F}^{b}\right)$, we need the following genericness assumption.

Setting 3.5. With the assumptions in Setting 3.2, we assume further that $n \geq b s-a+1$ and $\mathcal{F}=\left\{f_{1}, \ldots, f_{s}\right\}$ is a set of $(b s-a+1)$-generic $d$-forms in $R=\mathbb{K}\left[x_{0}, \ldots, x_{n}\right]$. 
From now on, we will fix a positive integer $m$ and investigate the symbolic power $I_{a}\left(\mathcal{F}^{b}\right)^{(m)}$. It follows from [17, Theoorem 4.6] that

$$
I_{a}\left(\mathcal{F}^{b}\right)^{(m)}=\bigcap_{c=c_{0}}^{h} I_{c, \mathcal{F}}^{\left(m\left(\mu_{a}^{0}+b\left(c-c_{0}\right)\right)\right)} .
$$

Inspired by the discussion in [8, Subsection 4.2], we simultaneously look at the ideals

$$
K_{j}:=\bigcap_{c=c_{0}}^{h-j} I_{c, \mathcal{F}}^{\left(m\left(\mu_{a}^{0}+b\left(c-c_{0}\right)\right)\right)} \quad \text { and } \quad K_{j}(\boldsymbol{z}):=\bigcap_{c=c_{0}}^{h-j} I_{c, \mathcal{F}_{\boldsymbol{z}}}^{\left(m\left(\mu_{a}^{0}+b\left(c-c_{0}\right)\right)\right)}
$$

for $j=0,1, \ldots, h-c_{0}$. We may also write $K_{h-c_{0}+1}=R$ and $K_{h-c_{0}+1}(\boldsymbol{z})=T$. Thus, we have a filtration of submodules

$$
0=\overline{I_{a}\left(\mathcal{F}^{b}\right)^{(m)}}=\overline{K_{0}} \subseteq \overline{K_{1}} \subseteq \cdots \subseteq \overline{K_{h-c_{0}}} \subseteq R / I_{a}\left(\mathcal{F}^{b}\right)^{(m)},
$$

where - means the corresponding image in $R / I_{a}\left(\mathcal{F}^{b}\right)^{(m)}$. And we have a similar filtration of submodules for $T / I_{a}\left(\mathcal{F}_{\boldsymbol{z}}^{b}\right)^{(m)}$. Now, $\overline{K_{j+1}(\boldsymbol{z})} / \overline{K_{j}(\boldsymbol{z})} \cong K_{j+1}(\boldsymbol{z}) / K_{j}(\boldsymbol{z})$ is either 0 or Cohen-Macaulay of expected dimension for each $j$, by [8, Theorem A.4] and Proposition 3.3. It remains to check if each $\overline{K_{j+1}} / \overline{K_{j}}$ still has the similar property.

Lemma 3.6. (a) If $\overline{K_{j+1}} \neq \overline{K_{j}}$, then $\operatorname{dim}(R)-\operatorname{dim}\left(\overline{K_{j+1}} / \overline{K_{j}}\right)=h-j$.

(b) Similarly, if $\overline{K_{j+1}(\boldsymbol{z})} \neq \overline{K_{j}(\boldsymbol{z})}$, then $\operatorname{dim}(T)-\operatorname{dim}\left(\overline{K_{j+1}(\boldsymbol{z})} / \overline{K_{j}(\boldsymbol{z})}\right)=h-j$ as well.

Proof. In the first case,

$$
\begin{aligned}
\overline{K_{j+1}} / \overline{K_{j}} & \cong K_{j+1} / K_{j}=K_{j+1} /\left(K_{j+1} \cap I_{c, \mathcal{F}}^{\left(m\left(\mu_{a}^{0}+b\left(c-c_{0}\right)\right)\right)}\right) \\
& \cong\left(K_{j+1}+I_{c, \mathcal{F}}^{\left(m\left(\mu_{a}^{0}+b\left(c-c_{0}\right)\right)\right)}\right) / I_{c, \mathcal{F}}^{\left(m\left(\mu_{a}^{0}+b\left(c-c_{0}\right)\right)\right)} \subset R / I_{c, \mathcal{F}}^{\left(m\left(\mu_{a}^{0}+b\left(c-c_{0}\right)\right)\right)}
\end{aligned}
$$

for $c=h-j$. Thus, $\operatorname{Ass}\left(\overline{K_{j+1}} / \overline{K_{j}}\right) \subseteq \operatorname{Ass}\left(R / I_{c, \mathcal{F}}^{\left(m\left(\mu_{a}^{0}+b\left(c-c_{0}\right)\right)\right)}\right)$. But $R / I_{c, \mathcal{F}}^{\left(m\left(\mu_{a}^{0}+b\left(c-c_{0}\right)\right)\right)}$ is Cohen-Macaulay of $\operatorname{dimension} \operatorname{dim}(R)-c$ by [12, Corollary 3.7]. This shows that $\operatorname{dim}\left(\overline{K_{j+1}} / \overline{K_{j}}\right)=\operatorname{dim}(R)-c$ as well. The second case is similar.

Lemma 3.7. Let $\varphi: T=\mathbb{K}\left[z_{1}, \ldots, z_{s}\right] \rightarrow R$ be the homomorphism induced by $z_{i} \mapsto f_{i}$ for each $i$. Suppose that $\boldsymbol{F}_{\boldsymbol{\bullet}}$ is a graded minimal free resolution of $T / K_{j}(\boldsymbol{z})$ for $\mathcal{F}_{\boldsymbol{z}}=$ $\left\{z_{1}, \ldots, z_{s}\right\} \subseteq T$. Then, $K_{j}=\varphi\left(K_{j}(\boldsymbol{z})\right) R$ and $\boldsymbol{F}_{\bullet} \otimes_{T} R$ is a graded minimal free resolution of $R / K_{j}$.

Proof. The argument will be similar to the proof of [12, Theorem 3.3]. We prove by induction on $s-\hat{c}$, where $\hat{c}:=\max \{c: \mathcal{F}$ is $c$-generic $\}$. Obviously, $h \leq \hat{c} \leq s-1$. If $s-\hat{c}=1, \mathcal{F}$ forms a regular sequence. Whence, $\varphi$ is flat and the claim is clear by [17, Remark 2.1] or [12, Lemma 3.1] and its proof.

When $s-\hat{c}>1$, let $y$ be a new variable over $R$. For each $i$, let $f_{i}^{\prime}$ be a general $d$-form in the ideal $\left(f_{i}, y\right) \subset R[y]$. Now, consider the new homomorphism $\gamma: T \rightarrow R[y]$ induced by $z_{i} \mapsto f_{i}^{\prime}$. Notice that $\mathcal{F}^{\prime}:=\left\{f_{1}^{\prime}, \ldots, f_{s}^{\prime}\right\}$ is a set of $(\hat{c}+1)$-generic forms in $R[y]$. Whence, by induction, $\boldsymbol{F}_{\bullet} \otimes_{T} R[y]$ is a graded minimal free resolution of $R[y] / K_{j}^{\prime}$ where $K_{j}^{\prime}=\bigcap_{c=c_{0}}^{h-j} I_{c, \mathcal{F}^{\prime}}^{\left(m\left(\mu_{a}^{0}+b\left(c-c_{0}\right)\right)\right)}$. Meanwhile, we have the graded isomorphism

$$
R[y] /\left(K_{j}^{\prime}, y\right) \cong R / K_{j} .
$$

Thus, the last piece of the proof is to show that $y$ is a non-zero-divisor of $R[y] / K_{j}^{\prime}$.

Suppose for contradiction that $y$ is not regular modulo the ideal $K_{j}^{\prime}$, then $y \in \mathfrak{p}$ for some associated prime $\mathfrak{p}$. Notice that this is equivalent to saying that $y$ is not regular 
when modulo $I_{c, \mathcal{F}^{\prime}}^{\left(m\left(\mu_{a}^{0}+b\left(c-c_{0}\right)\right)\right)}$ for some $c$ with $c_{0} \leq c \leq h-j$. But this is impossible by the proof of [12, Theorem 3.3].

Here is the main result of this section.

Theorem 3.8. Assuming Setting 3.5, the symbolic powers of $I_{a}\left(\mathcal{F}^{b}\right)=I_{a}\left(f_{1}^{b} \cdots f_{s}^{b}\right)$ are all sequentially Cohen-Macaulay.

Proof. We will fix a positive integer $m$ and look at the symbolic power $I_{a}\left(\mathcal{F}^{b}\right)^{(m)}$. After applying the notations $K_{j}$ and $K_{j}(\boldsymbol{z})$ introduced before Lemma 3.6, we have a short exact sequence

$$
0 \rightarrow K_{j+1}(\boldsymbol{z}) / K_{j}(\boldsymbol{z}) \rightarrow T / K_{j}(\boldsymbol{z}) \rightarrow T / K_{j+1}(\boldsymbol{z}) \rightarrow 0 .
$$

We may assume that $K_{j} \neq K_{j+1}$. Hence, by Lemma 3.7, $K_{j}(\boldsymbol{z}) \neq K_{j+1}(\boldsymbol{z})$. Furthermore,

$$
\operatorname{Tor}_{k}^{T}\left(T / K_{j}(\boldsymbol{z}), R\right)=0=\operatorname{Tor}_{k}^{T}\left(T / K_{j+1}(\boldsymbol{z}), R\right)
$$

for $k \geq 1$. If we take the well-known induced long exact sequence, then we obtain $\operatorname{Tor}_{k}^{T}\left(K_{j+1}(\boldsymbol{z}) / K_{j}(\boldsymbol{z}), R\right)=0$ as well for all $k \geq 1$. Furthermore, since $\left(T / K_{j}(\boldsymbol{z})\right) \otimes R \cong$ $R / K_{j},\left(T / K_{j+1}(\boldsymbol{z})\right) \otimes R \cong R / K_{j+1}$ and $\operatorname{Tor}_{1}\left(T / K_{j+1}(\boldsymbol{z}), R\right)=0$, we see immediately that $\left(K_{j+1}(\boldsymbol{z}) / K_{j}(\boldsymbol{z})\right) \otimes R \cong K_{j+1} / K_{j}$. Therefore, if $\boldsymbol{G}_{\bullet}$ is a graded minimal free resolution of $K_{j+1}(\boldsymbol{z}) / K_{j}(\boldsymbol{z})$, then $\boldsymbol{G}_{\bullet} \otimes R$ will be a graded minimal free resolution of $K_{j+1} / K_{j}$. In particular, $\operatorname{pd}\left(K_{j+1} / K_{j}\right)=\operatorname{pd}\left(K_{j+1}(\boldsymbol{z}) / K_{j}(\boldsymbol{z})\right)$.

Since $K_{j+1}(\boldsymbol{z}) / K_{j}(\boldsymbol{z})$ is Cohen-Macaulay, it follows from Lemma 3.6 and the AuslanderBuchsbaum formula that $K_{j+1} / K_{j}$ is also Cohen-Macaulay of the expected dimension. Therefore, $I_{a}\left(\mathcal{F}^{b}\right)^{(m)}$ is sequentially Cohen-Macaulay.

\section{Harbourne-Huneke Containment problem For the Uniform $a$-FOld PRODUCT IDEAL}

Recall that for any homogeneous ideal $I$ in a standard graded ring, $\alpha(I)$ stands for the least degree of a homogeneous polynomial in this ideal. This final section is mainly concerned with the following two questions involving this invariant, which were raised for radical homogeneous ideals in [4, Section 3].

Question 4.1. [Demailly-like bound] Let $R$ be a polynomial ring over a field $\mathbb{K}$ and let $I$ be a homogeneous ideal of big height $h$ in $R$. Does the inequality

$$
\frac{\alpha\left(I^{(n)}\right)}{n} \geq \frac{\alpha\left(I^{(m)}\right)+h-1}{m+h-1}
$$

hold for all $n, m \geq 1$ ?

Notice that the Waldschmidt constant

$$
\widehat{\alpha}(I):=\lim _{n} \frac{\alpha\left(I^{(n)}\right)}{n}
$$

actually agrees with $\inf _{n} \frac{\alpha\left(I^{n}\right)}{n}$. Thus, the above inequality is simply

$$
\widehat{\alpha}(I) \geq \frac{\alpha\left(I^{(m)}\right)+h-1}{m+h-1} .
$$

Question 4.2. [General version of the Harbourne-Huneke containment for radical homogeneous ideal] Let $R$ be a polynomial ring over a field $\mathbb{K}$ with the maximal homogeneous ideal $\mathfrak{m}$. If $I$ is a homogeneous ideal of big height $h$ in $R$, does the inequality

$$
I^{(\ell(h+m-1))} \subseteq \mathfrak{m}^{\ell(h-1)}\left(I^{(m)}\right)^{\ell}
$$

holds for all $\ell, m \geq 1$ ? 
We will drop the radical requirement and study these questions for $I=I_{a}\left(\mathcal{F}^{b}\right)$ under the assumptions in Setting 3.5. It is not difficult to see that a positive answer to Question 4.2 would imply an affirmative answer to Question 4.1. Thus, we will focus on Question 4.2. Actually, like [4, Theorem 3.6], a stronger result can be achieved. Notice that the big height of the ideal $I_{a}\left(\mathcal{F}^{b}\right)$ is $h=b s-a+1$ while the height is the positive integer $c_{0}$ by [17, Remark 4.5]. Furthermore, we have seen in the proof of [17, Proposition 4.7] that $c_{0}=h$ precisely when $b=1$ or $a=b s$. The $b=1$ case is just the standard star configuration case studied in [4]. Meanwhile, the $a=b s$ case is the trivial one when the ideal is actually principal. Thus, in the following, we may assume that $c_{0}<h$. Consequently, $b>1, a<b s$ and $h-1>0$.

We present two technical lemmas first.

Lemma 4.3. We have $(h-1)\left(\mu_{a}^{0}+b\left(c-c_{0}\right)\right) \geq c$ for each $c$ with $c_{0} \leq c \leq h$.

Proof. Notice that the coefficients of $c$ are $(h-1) b$ and 1 on the two sides. Since $(h-1) b \geq$ 1 , it suffices to show the inequality for $c=c_{0}$, namely, to show

$$
(h-1) \mu_{a}^{0} \geq c_{0} .
$$

After putting back the definitions of $h, \mu_{a}^{0}$ and $c_{0}$, this inequality is actually

$$
(b s-a)\left(a-b\left\lfloor\frac{a-1}{b}\right\rfloor\right) \geq s-\left\lfloor\frac{a-1}{b}\right\rfloor .
$$

And it can be simplified into

$$
(b s-a) a-s \geq((b s-a) b-1)\left\lfloor\frac{a-1}{b}\right\rfloor .
$$

Since $b s>a$ and $b>1$, we have $(b s-a) b-1>0$. Thus, it suffices to show that

$$
(b s-a) a-s \geq((b s-a) b-1) \cdot \frac{a-1}{b},
$$

which is equivalent to saying that

$$
(b-1)(b s-a) \geq 1 .
$$

However, the last inequality is clear. Thus, we have successfully shown the inequality (17). And this completes our proof for Lemma 4.3.

In the following, for each positive integer $c$ such that $c_{0} \leq c \leq h$, we write $\widehat{\mu}_{c}$ for the positive integer $\mu_{a}^{0}+b\left(c-c_{0}\right)$.

Lemma 4.4. Let $k, \ell$, and $m$ be positive integers. Then, we have

$$
(\ell(h+m-1)-h+k) \widehat{\mu}_{c}-c(\ell-1) \geq \ell m \widehat{\mu}_{c}
$$

for each $c$ with $c_{0} \leq c \leq h$.

Proof. This artificially irregular inequality can be quickly modified into

$$
\ell\left((h-1) \widehat{\mu}_{c}-c\right) \geq(h-k) \widehat{\mu}_{c}-c .
$$

Since $(h-1) \widehat{\mu}_{c} \geq c$ by Lemma 4.3, we only need to show (8) for $\ell=1$, i.e., to show that

$$
(h-1) \widehat{\mu}_{c}-c \geq(h-k) \widehat{\mu}_{c}-c .
$$

However, this holds automatically, since both $k$ and $\widehat{\mu}_{c}$ are positive integers.

Now, we are ready for the main theorem of this section. 
Theorem 4.5. Under the assumptions in Setting 3.5, the ideal $I=I_{a}\left(\mathcal{F}^{b}\right)$ satisfies

$$
I^{(\ell(h+m-1)-h+k)} \subseteq \mathfrak{m}^{d((\ell-1)(h-1)+k-1) \widehat{\mu}_{h}}\left(I^{(m)}\right)^{\ell}
$$

for all positive integers $k, \ell$ and $m$.

Note that $\widehat{\mu}_{h}$ above is simply $\mu_{a}^{0}+b\left(h-c_{0}\right)=b h-(b s-a)$.

Proof. Firstly, we consider the standard monomial case when $I=I_{a}\left(\mathcal{F}_{z}^{b}\right)$ for $\mathcal{F}_{\boldsymbol{z}}=$ $\left\{z_{1}, \ldots, z_{s}\right\}$ in $T=\mathbb{K}\left[z_{1}, \ldots, z_{s}\right]$. Within this ring, by abuse of notation, we will write $\boldsymbol{z}^{\boldsymbol{\mu}}:=z_{1}^{\mu_{1}} \cdots z_{s}^{\mu_{s}}$ for any tuple $\boldsymbol{\mu}=\left(\mu_{1}, \ldots, \mu_{s}\right) \in \mathbb{Z}_{\geq 0}^{s}$. As usual, $|\boldsymbol{\mu}|$ is simply $\mu_{1}+\cdots+\mu_{s}$, and $\boldsymbol{\mu}_{\leq c}$ is short for the sub-tuple $\left(\mu_{1}, \ldots, \mu_{c}\right)$. Since the permutation group $\mathfrak{S}_{s}$ has a natural action on $T$, for any monomial ideal $K$ that is invariant under this action, we will introduce

$$
\mathcal{P}(K):=\left\{\boldsymbol{\lambda} \text { is a partition of length } s: \boldsymbol{z}^{\boldsymbol{\lambda}} \in K\right\}
$$

and, by abuse of notation,

$$
\Lambda(K):=\left\{\boldsymbol{\lambda}: \boldsymbol{z}^{\boldsymbol{\lambda}} \text { is a minimal monomial generator of } K\right\} .
$$

Take an arbitrary partition $\boldsymbol{\lambda}=\left(\lambda_{1}, \ldots, \lambda_{s}\right) \in \mathcal{P}\left(I_{a}\left(\mathcal{F}_{\boldsymbol{z}}^{b}\right)^{\left(m^{\prime}\right)}\right)$ where $m^{\prime}:=\ell(h+m-$ $1)-h+k$. Due to the decomposition in (6), this amounts to saying that

$$
\boldsymbol{\lambda} \in \mathcal{P}\left(I_{c, \mathcal{F}_{\boldsymbol{z}}}^{\left(m^{\prime} \widehat{\mu}_{c}\right)}\right) \quad \text { for each } c \text { with } c_{0} \leq c \leq h,
$$

namely,

$$
\left|\boldsymbol{\lambda}_{\leq c}\right| \geq m^{\prime} \widehat{\mu}_{c} \quad \text { for each } c \text { with } c_{0} \leq c \leq h
$$

by [3, Proposition 4.1].

In the following, for each $j \leq h$, we write $\lambda_{j}^{\prime}:=\left\lfloor\frac{\lambda_{j}}{\ell}\right\rfloor$ and $\widehat{\lambda}_{j}:=\lambda_{j}-\ell \lambda_{j}^{\prime}$. Since $\widehat{\lambda}_{j} \leq \ell-1$ for such $j$, we have $\sum_{j \leq c} \widehat{\lambda}_{j} \leq c(\ell-1)$. Thus,

$$
\sum_{j \leq c} \ell \lambda_{j}^{\prime}=\sum_{j \leq c}\left(\lambda_{j}-\widehat{\lambda}_{j}\right)=\sum_{j \leq c} \lambda_{j}-\sum_{j \leq c} \widehat{\lambda}_{j} \geq m^{\prime} \widehat{\mu}_{c}-c(\ell-1) .
$$

As a quick consequence of Lemma 4.4, we have

$$
\sum_{j \leq c} \lambda_{j}^{\prime} \geq m \widehat{\mu}_{c}
$$

for each such $c$. It follows again from [3, Proposition 4.1] and the decomposition in (6) that

$$
\boldsymbol{\lambda}^{\prime}:=\left(\lambda_{1}^{\prime}, \lambda_{2}^{\prime}, \ldots, \lambda_{h}^{\prime}, \lambda_{h}^{\prime}, \ldots, \lambda_{h}^{\prime}\right) \in \mathcal{P}\left(I_{a}\left(\mathcal{F}_{\boldsymbol{z}}^{b}\right)^{(m)}\right) .
$$

Therefore, we can find suitable partition $\boldsymbol{\lambda}^{\prime \prime}=\left(\lambda_{1}^{\prime \prime}, \ldots, \lambda_{s}^{\prime \prime}\right) \in \Lambda\left(I_{a}\left(\mathcal{F}_{\boldsymbol{z}}^{b}\right)^{(m)}\right)$ such that $\boldsymbol{\lambda}^{\prime \prime} \leq \boldsymbol{\lambda}^{\prime}$ componentwise. In the part (b) of the proof of [17, Proposition 4.7], we have shown that $\left|\boldsymbol{\lambda}_{\leq h}^{\prime \prime}\right|=m \widehat{\mu}_{h}$. Furthermore, $\lambda_{j}^{\prime \prime}=\lambda_{h}^{\prime \prime}$ whenever $h \leq j \leq s$. Now, $\ell \boldsymbol{\lambda}^{\prime \prime} \leq \boldsymbol{\lambda}$ componentwise. And it remains to estimate $|\boldsymbol{\lambda}|-\ell\left|\boldsymbol{\lambda}^{\prime \prime}\right|$. It is clear that

$$
\begin{aligned}
|\boldsymbol{\lambda}|-\ell\left|\boldsymbol{\lambda}^{\prime \prime}\right| & =\sum_{j=1}^{s}\left(\boldsymbol{\lambda}_{j}-\ell \boldsymbol{\lambda}_{j}^{\prime \prime}\right) \geq \sum_{j=1}^{h}\left(\boldsymbol{\lambda}_{j}-\ell \boldsymbol{\lambda}_{j}^{\prime \prime}\right)=\left|\boldsymbol{\lambda}_{\leq h}\right|-\ell\left|\boldsymbol{\lambda}_{\leq h}^{\prime \prime}\right| \\
& \geq m^{\prime} \widehat{\mu}_{h}-\ell m \widehat{\mu}_{h}=(\ell(h+m-1)-h+k-\ell m) \widehat{\mu}_{h} \\
& =((\ell-1)(h-1)+k-1) \widehat{\mu}_{h} .
\end{aligned}
$$

Consequently, for $I=I_{a}\left(\mathcal{F}_{z}^{b}\right)$, we have

$$
I^{(\ell(h+m-1)-h+k)} \subseteq \mathfrak{m}^{((\ell-1)(h-1)+k-1) \widehat{\mu}_{h}}\left(I^{(m)}\right)^{\ell},
$$

establishing the containment (9) in this standard monomial case. 
In the final step, we consider the general uniform star configuration case, namely when $I=I_{a}\left(\mathcal{F}^{b}\right)$. Notice that both $I^{(\ell(h+m-1)-h+k)}$ and $I^{(m)}$ are generated by monomials in the forms in $\mathcal{F}$; cf. [17, Proposition 4.11]. Thus, it follows directly from (10) that

$$
I^{(\ell(h+m-1)-h+k)} \subseteq \mathfrak{m}^{d((\ell-1)(h-1)+k-1) \widehat{\mu}_{h}}\left(I^{(m)}\right)^{\ell},
$$

where $d$ is the common degree of the forms in $\mathcal{F}$.

The following result generalizes [4, Corollary 3.7].

Corollary 4.6. The Harbourne-Huneke type containment (Question 4.2) and the Demaillylike bound (Question 4.1) hold for the ideal $I=I_{a}\left(\mathcal{F}^{b}\right)$ under the assumptions in Setting 3.5 .

Proof. The Harbourne-Huneke type containment follows from (9), since both $d$ and $\widehat{\mu}_{h}$ are positive integers, while $k$ can be specially taken to be $h$. By applying the identical arguments in the proof of [4, Corollary 3.7], we see that the Demailly-like bound also holds.

Acknowledgment. The authors are grateful to the software system Macaulay2 [13], for serving as an excellent source of inspiration. The second author is partially supported by the "Anhui Initiative in Quantum Information Technologies" (No. AHY150200).

\section{REFERENCES}

[1] A. Almousa, G. Fløystad, and H. Lohne, Polarizations of powers of graded maximal ideals (2020), available at arXiv.1912.03898

[2] B. Anzis, M. Garrousian, and Ş. O. Tohăneanu, Generalized star configurations and the Tutte polynomial., J. Algebr. Comb. 46 (2017), 165-187.

[3] J. Biermann, H. de Alba, F. Galetto, S. Murai, U. Nagel, A. O’Keefe, T. Römer, and A. Seceleanu, Betti numbers of symmetric shifted ideals, J. Algebra 560 (2020), 312-342.

[4] S. Bisui, E. Grifo, H. T. Hà, and T. T. Nguyên, Demailly's conjecture and the containment problem, available at arXiv:2009.05022.

[5] C. Bocci and B. Harbourne, Comparing powers and symbolic powers of ideals, J. Algebraic Geom. 19 (2010), 399-417.

[6] E. Carlini, L. Chiantini, and A. V. Geramita, Complete intersections on general hypersurfaces, Michigan Math. J. 57 (2008), 121-136.

[7] E. Carlini, E. Guardo, and A. Van Tuyl, Star configurations on generic hypersurfaces, J. Algebra 407 (2014), 1-20.

[8] S. Faridi, Monomial ideals via square-free monomial ideals, Commutative algebra, 2006, pp. 85-114.

[9] F. Galetto, On the ideal generated by all squarefree monomials of a given degree, J. Commut. Algebra 12 (2020), 199-215.

[10] M. Garrousian, A. Simis, and Ş. O. Tohăneanu, A blowup algebra for hyperplane arrangements, Algebra Number Theory 12 (2018), 1401-1429.

[11] A. V. Geramita, B. Harbourne, and J. Migliore, Star configurations in $\mathbb{P}^{n}$, J. Algebra 376 (2013), $279-299$.

[12] A. V. Geramita, B. Harbourne, J. Migliore, and U. Nagel, Matroid configurations and symbolic powers of their ideals, Trans. Amer. Math. Soc. 369 (2017), 7049-7066.

[13] D. R. Grayson and M. E. Stillman, Macaulay2, a software system for research in algebraic geometry. Available at http://www.math.uiuc.edu/Macaulay2/.

[14] B. Harbourne and C. Huneke, Are symbolic powers highly evolved?, J. Ramanujan Math. Soc. 28A (2013), 247-266.

[15] J. Herzog and T. Hibi, Monomial ideals, Graduate Texts in Mathematics, vol. 260, Springer-Verlag London Ltd., London, 2011.

[16] M. Hochster and C. Huneke, Comparison of symbolic and ordinary powers of ideals, Invent. Math. 147 (2002), 349-369.

[17] K.-N. Lin and Y.-H. Shen, Symbolic powers and free resolutions of generalized star configurations of hypersurfaces, accepted to the Michigan Math. J., available at arXiv:1912.04448. 
[18] P. Mantero, The structure and free resolution of the symbolic powers of star configurations of hypersurfaces, Trans. Amer. Math. Soc. 373 (2020), 8785-8835.

[19] Ş. O. Tohăneanu and Y. Xie, On the Geramita-Harbourne-Migliore conjecture, Trans. Amer. Math. Soc. 374 (2021), 4059-4073.

Department of Mathematics, The Penn State University, McKeesport, PA, 15132, USA Email address: kul20@psu.edu

Cas Wu Wen-Tsun Key Laboratory of Mathematics, School of Mathematical Sciences, University of Science and Technology of China, Hefei, Anhui, 230026, P.R. China Email address: yhshen@ustc.edu.cn 